VITAMIN D PROMOTES MYOGENIC DIFFERENTIATION AND INDUCES AN

ANTIFIBROTIC PHENOTYPE IN PRIMARY CULTURES OF SKELETAL MUSCLE DERIVED SATELLITE CELLS AND FIBROBLASTS

Amanda Daoud ${ }^{1}$, Irene Kim¹', Keith C. Norris ${ }^{3}$, Monica G. Ferrini11,2,3 and Jorge N. Artaza1,2,3 1Department of Health \& Life Sciences and '2Department of Internal Medicine, CDU, Los Angeles, CA, USA.
3Department of Medicine, David Geffen School of Medicine at UCLA, Los Angeles, CA, USA.

\section{Background I}

$\diamond$ Skeletal muscle wasting is a serious public health problem associated with aging, Chronic Kidney Disease (CKD), and AIDS'.

$\diamond$ Vitamin $\mathrm{D}(\mathrm{VD})$ is most widely recognized for its regulation of calcium and phosphate homeostasis in relation to bone development and maintenance, and for its synergistic effects on target organs such as PTH glands.

Recently, it has been shown to improve muscle performance and reduce falls in VD deficient older adults ${ }^{2,3}$. However, little is known of the underlying molecular mechanism or the role it plays in association with myogenic differentiation and on muscle fibrosis.

\section{Materials \& Methods}

Primary cultures of skeletal muscle derived satellite cells and fibroblasts were isolated from the tibialis anterior, soleus and gastrocnemius muscles of 2-month-old C57/ BL6 male mice and then treated with or without $1,25-D_{3}$ in a time course manner.

$\diamond$ Expression of Vitamin D receptor (VDR), collagen I, III, pro and anti-fibrotic factors, muscle lineage and angiogenic markers were assessed by Immunocytochemistry (ICC), PCR arrays and confirmed by Real time qPCR and western blots.

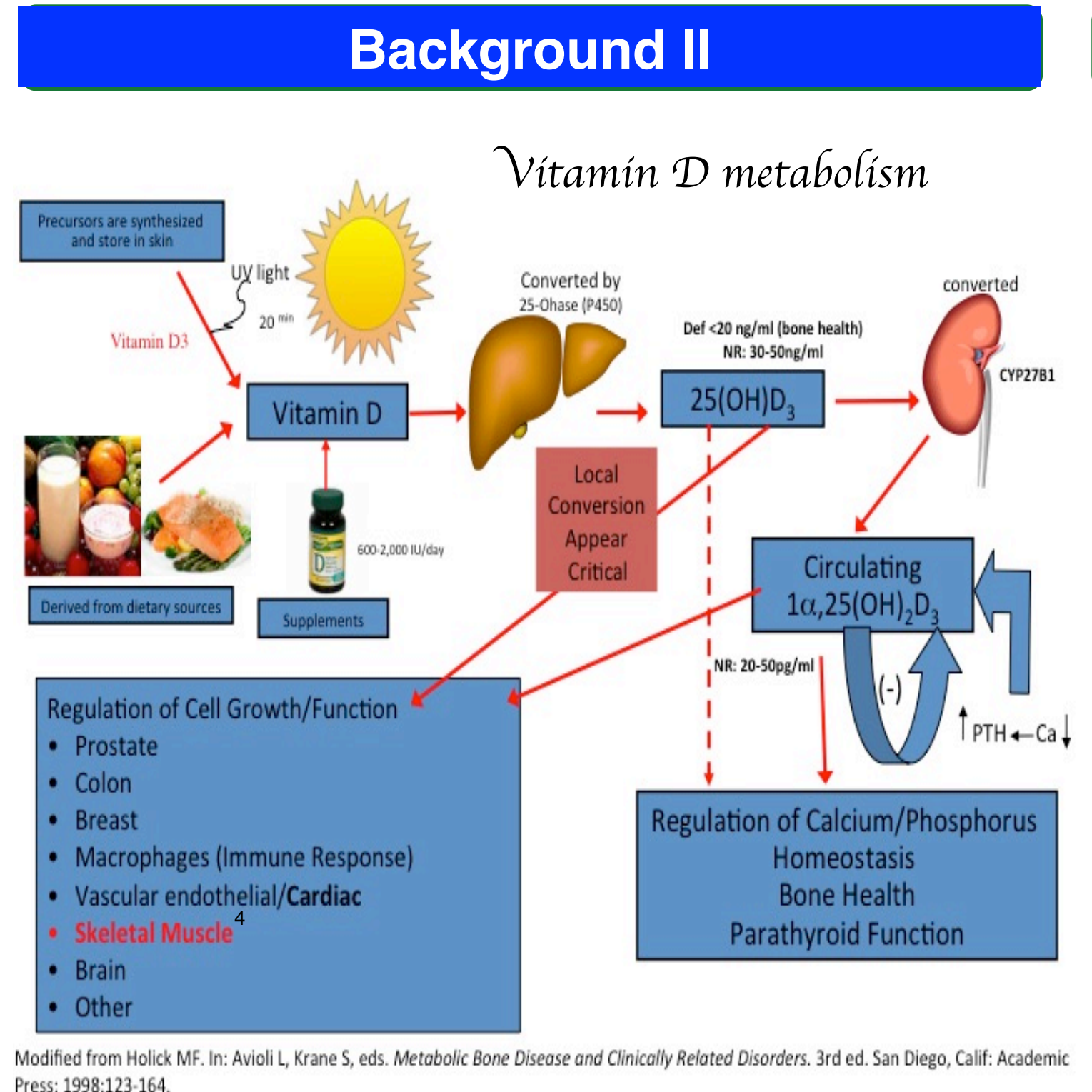

\section{Objectives}

$\diamond$ To examine the effects of $1,25-D_{3}$, the active form of Vitamin D, also known as 'calcitriol' on:

Myogenic cell differentiation of muscle stem cells (satellite cells), and

$>$ The generation of an anti-fibrotic phenotype in skeletal muscle derived fibroblast cells.

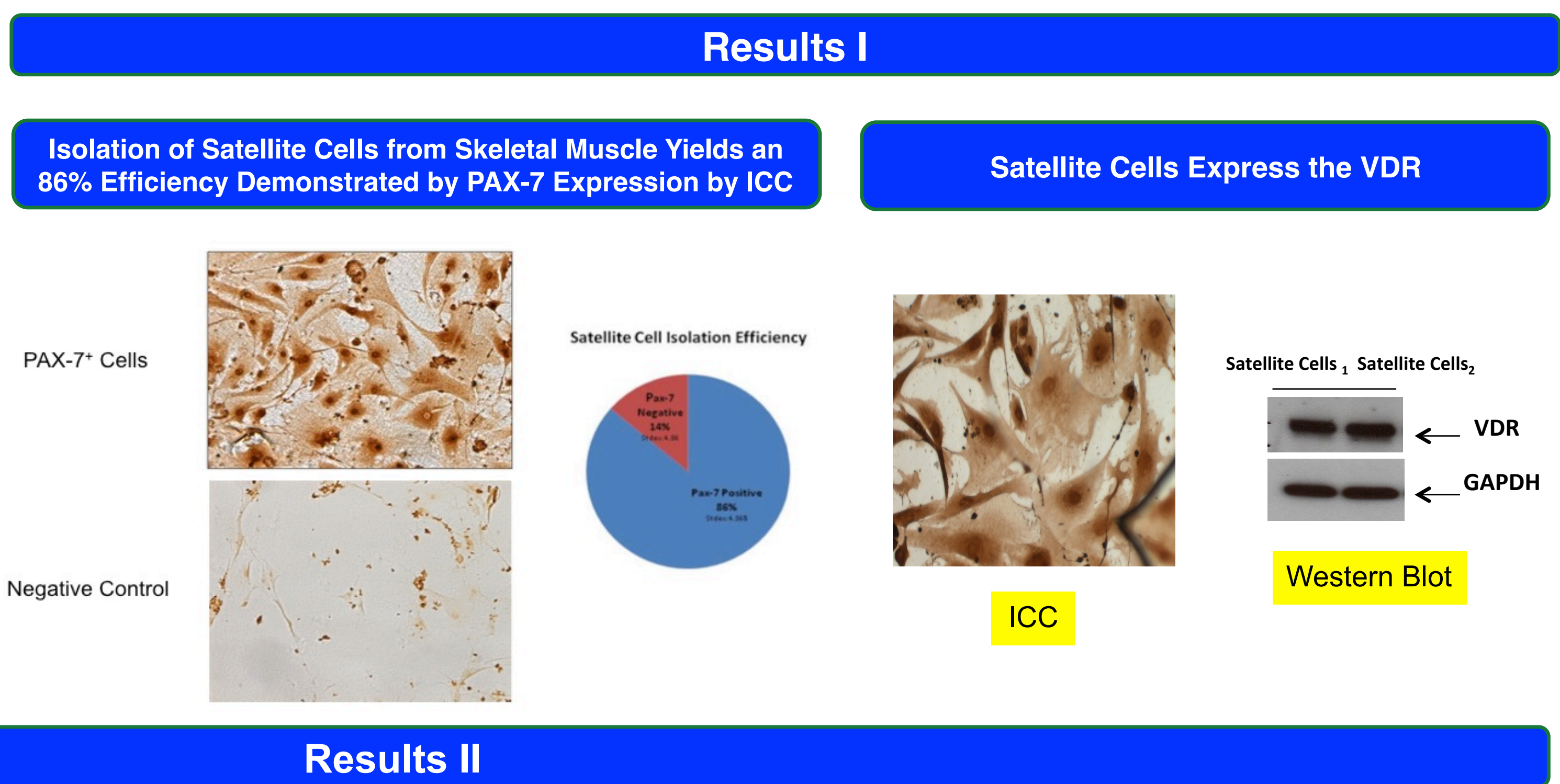

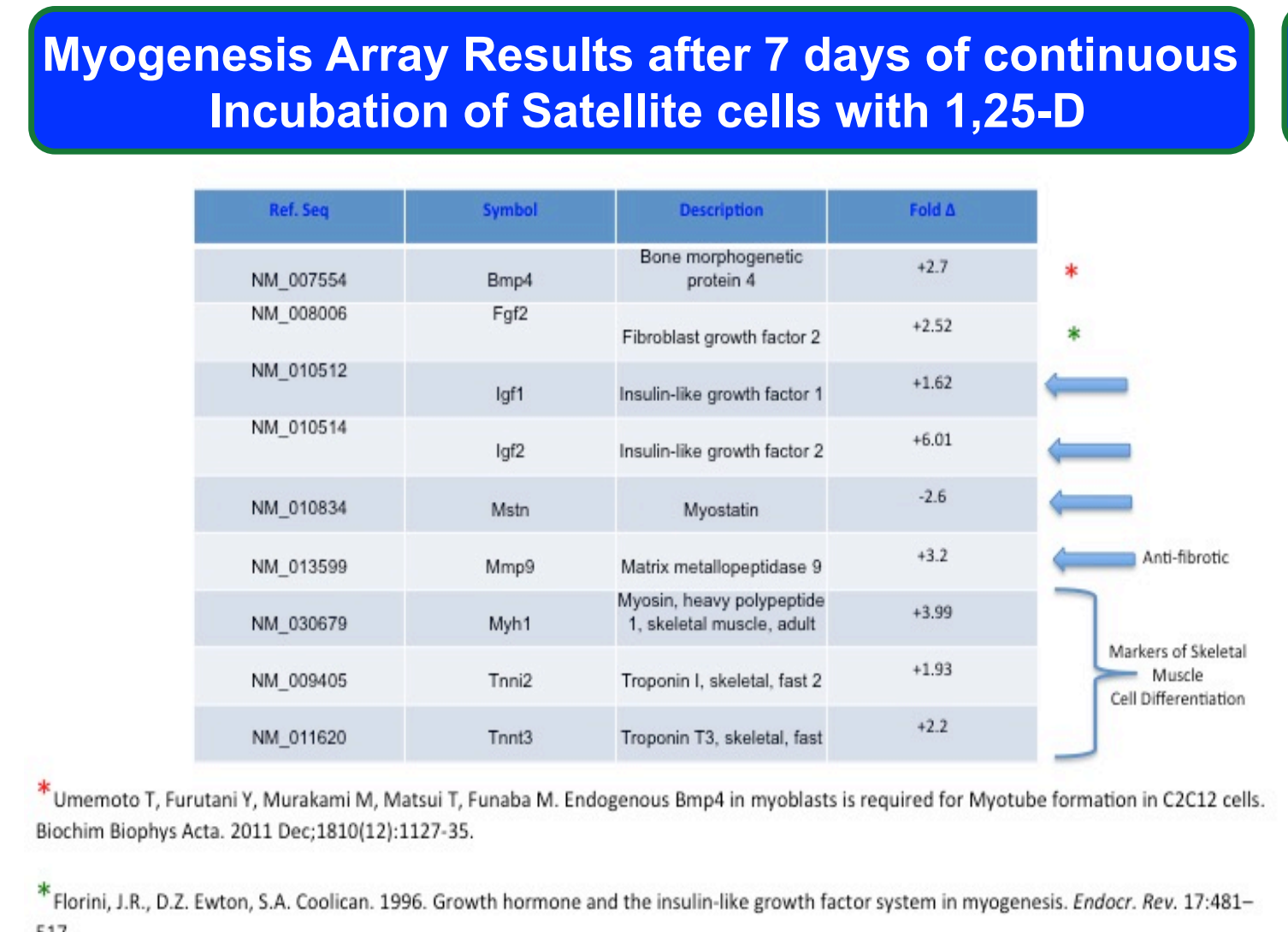

Characterization of Primary Cultures of Fibroblasts from Skeletal Muscle

$\checkmark$ Isolation of fibroblasts:

Cells were allowed to adhere for $2 \mathrm{~h}$ to remove large debris, macrophages and fibroblasts that adhere to the plastic, the non-adherent cells (satellite cells) were transfer to another flas.

Fibroblast were detached with a cell scraper and transfer to 6 -well plates for

characterization.
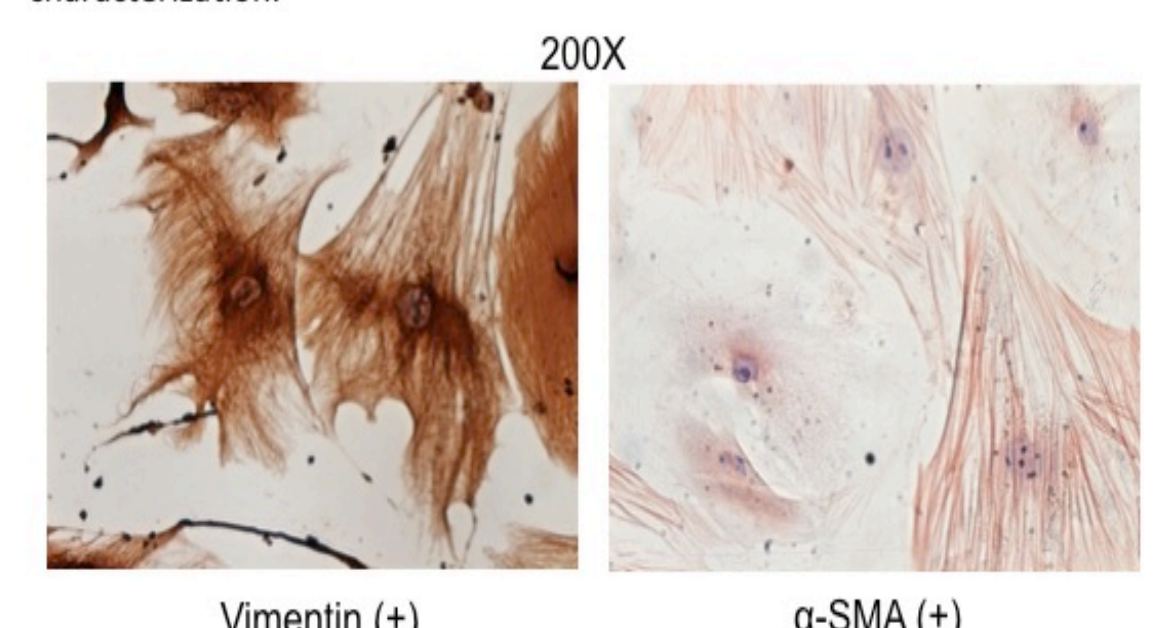

Vimentin (+)

a-SMA (+)

Conclusions

The efficiency of satellite cells isolation determined by PAX $-7^{+}$cells was $86 \%$

$\diamond$ It was confirmed that satellite cells expressed VDR $>$ Addition of $1,25-\mathrm{D}_{3}(100 \mathrm{nM})$ to satellite cells induces:

a) Increase expression of Troponin-I and II,

b) Increase expression of Bmp4

c) Increase expression of IGF-I and IGF-II,

d) Increase expression of Follistatin (Myostatin inhibitor)

e) A decrease expression of Mstn (Myostatin- a key negative regulator of muscle mass).

Fibroblast isolated with a $90 \%$ efficiency were characterized by Vimentin ${ }^{+}$and $\mathrm{a}-\mathrm{SMA}^{+}$cells showed a decreased expression of collagen I and III after being challenged with TGF- $\beta$ alone or in combination with $1,25-D_{3}$
Expression of IGF-1 and IGF-2 by Real Time PCR

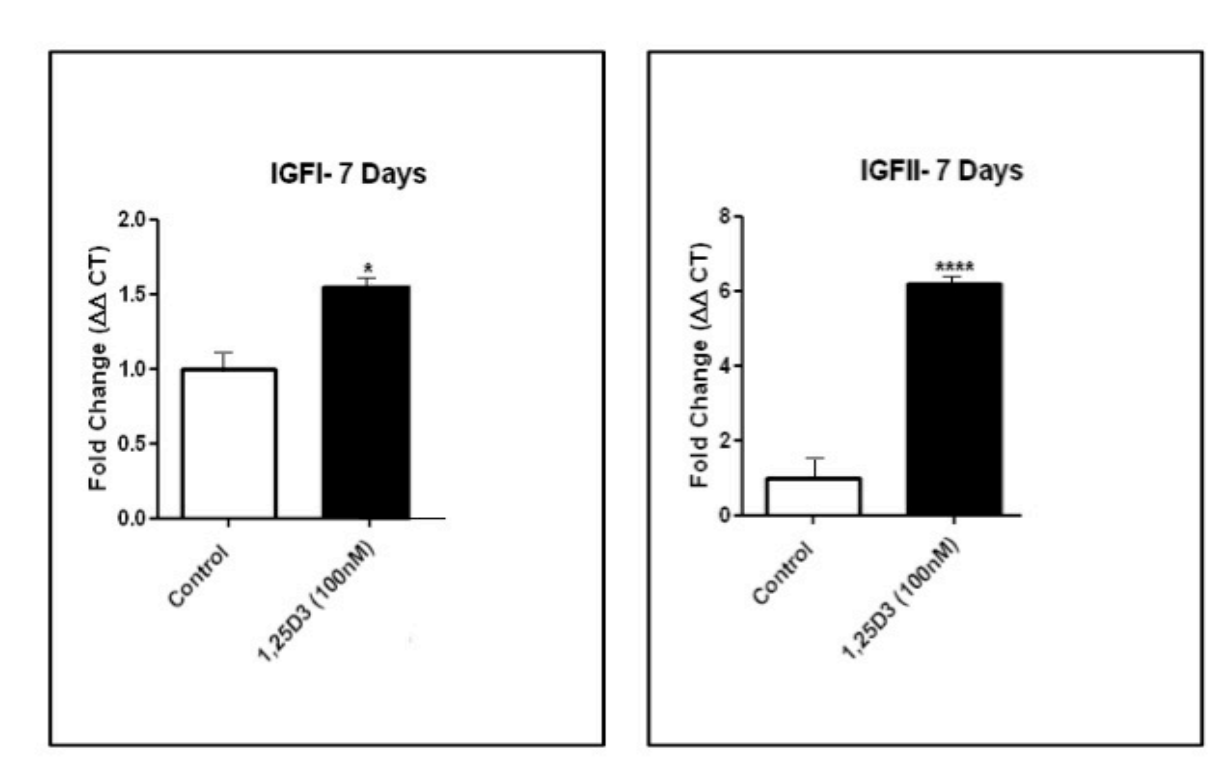

Vitamin D decreases the Expression of Collagen I in

primary cultures of Fibroblast treated with TGF- $\beta$

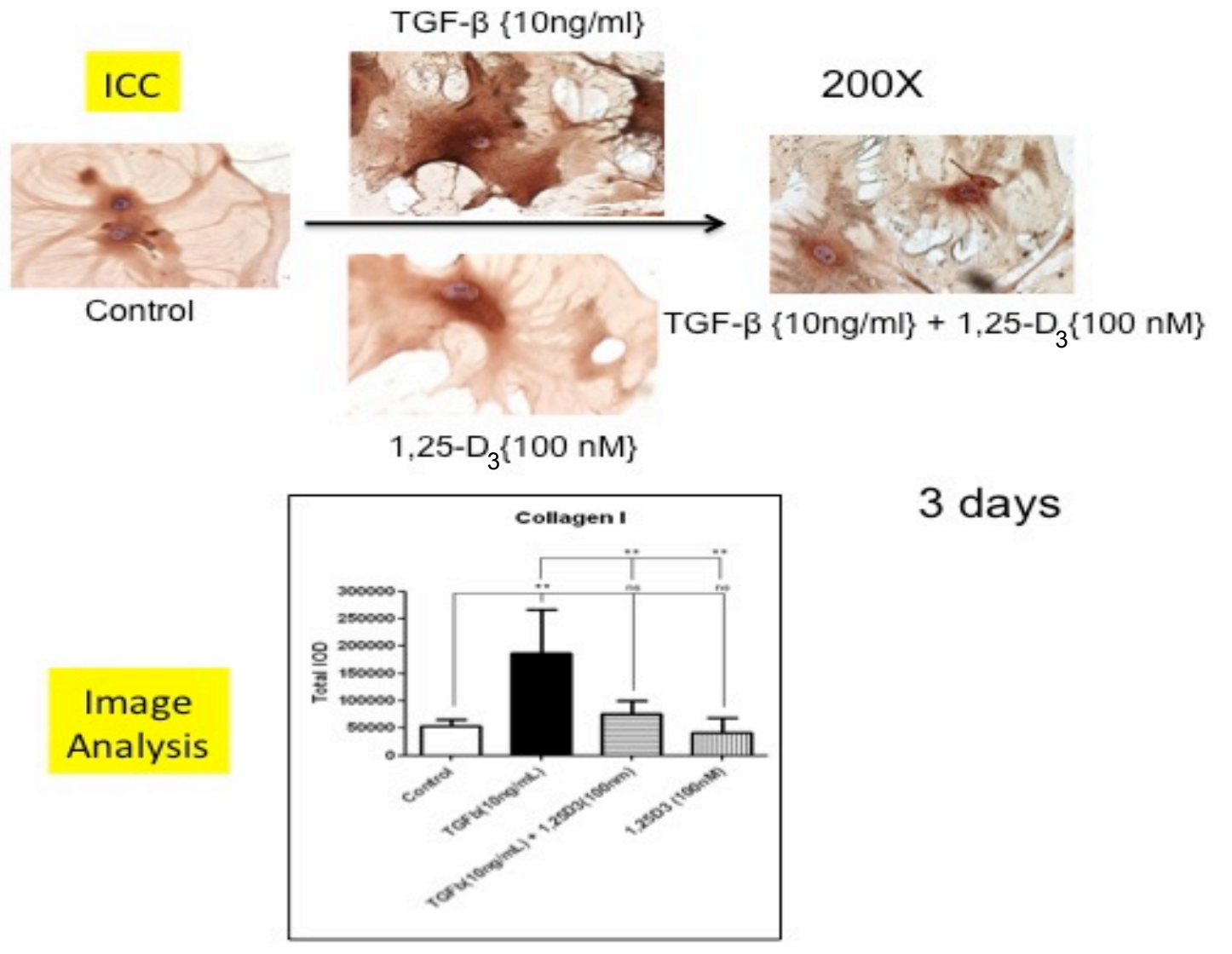

\section{summay}

Vitamin $D$ possesses a clear myogenic effect on satellite cells (adult muscle stem cells) in charge of reconstituting the muscle after muscle injury or muscle waste.

Vitamin $D$ also possesses an anti-fibrotic effect on fibroblasts of muscle origin.

This study provides a mechanistic justification for Vitamin $D$ replenishment in

Muscle waste conditions such as: AIDS, cancer congestive heart failure and renal failure characterized by lost of muscle mass and excessive collagen deposition (fibrotic process) and also in

Vitamin $D$ deficient older adults who are known to have age-related loss of muscle mass and an increased rate of falls.
Expression of Mstn and Fst by Real Time PCR and Western Blots
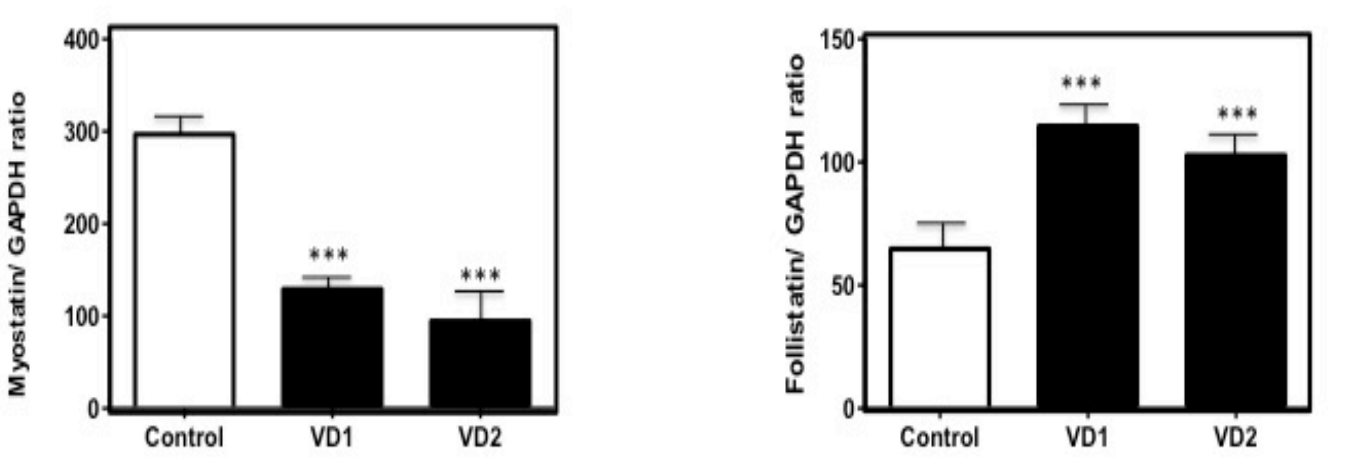

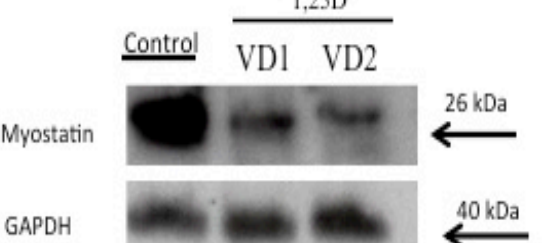

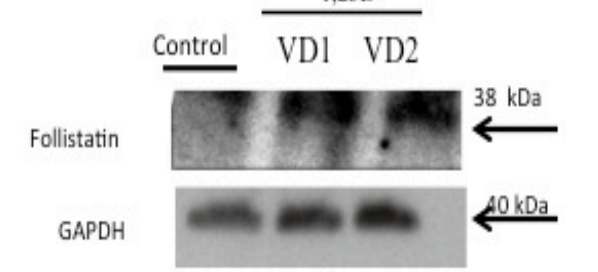

Vitamin D decreases the Expression of Collagen III in primary cultures of Fibroblast treated with TGF- $\beta$
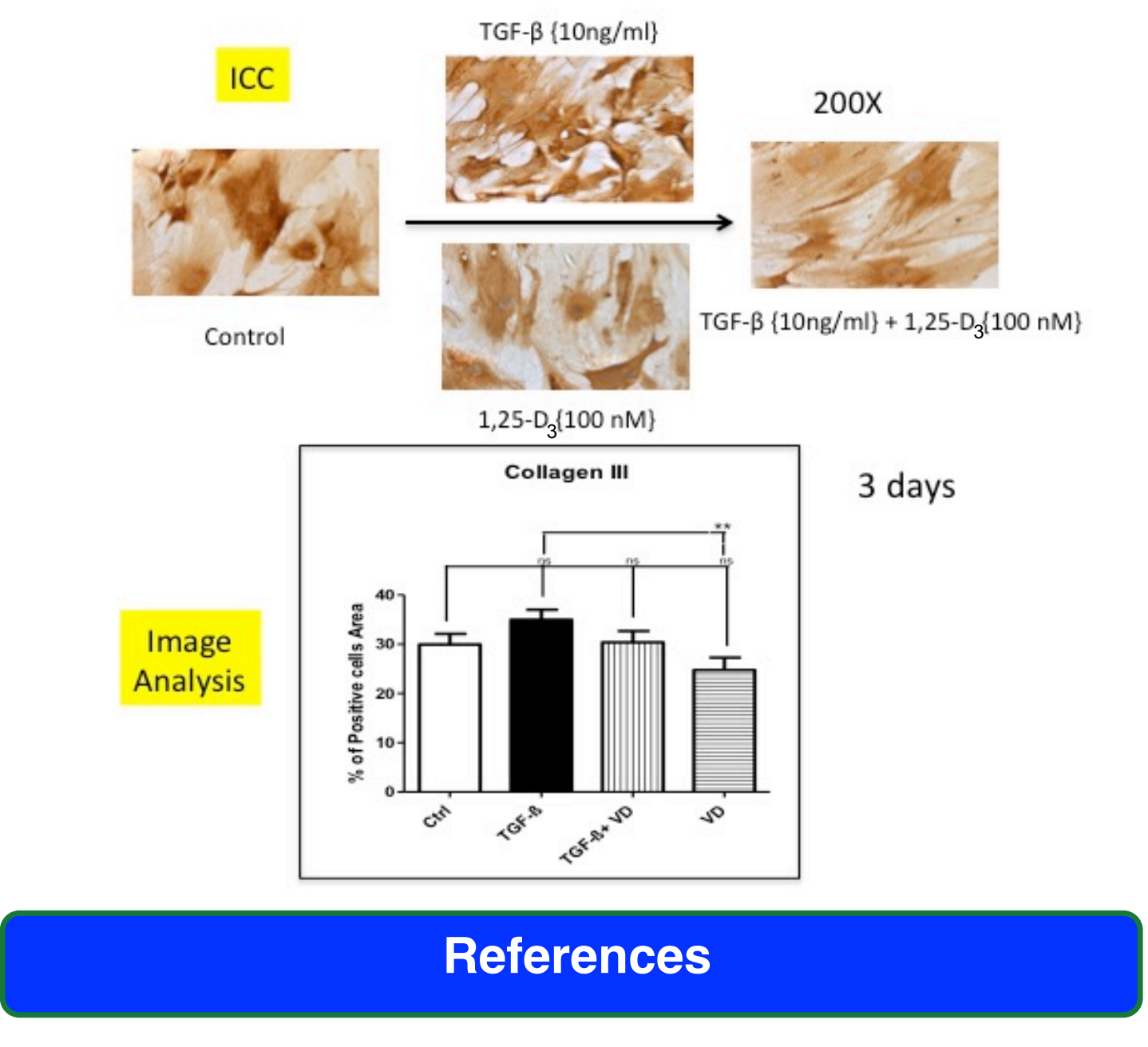

Holick MF. 2006. The role of vitamin D for bone health and fracture prevention. Curr Osteoporos Rep 4:96-102.

Glerup H, Mikkelsen K, Poulsen L, Hass E, Overbeck S, Andersen H, Charles P, Eriksen EF. 2000. Hypovitaminosis myopathy whout biochemical Eigns of

Bordelon P, Ghetu MV, Langan RC. 2009. Recognition and management of vitamin $\mathrm{D}$ deficiency. Am Fam Physician 80:841-846.

Garcia LA, King KK, Ferrini MG, Norris KC, Artaza JN $1,25(\mathrm{OH})$ 2vitamin D3 stimulates myogenic differentiation by inhibiting cell proliferation and modulating the expression promyogenic growth factors and myostatin in $\mathrm{C} 2 \mathrm{C} 12$ skeleta muscle cells. Endocrinology. 2011 Aug;152(8):2976-86. 